\title{
Genome-wide Chromatin Profiling of Legionella pneumophila-Infected Human Macrophages Reveals Activation of the Probacterial Host Factor TNFAIP2
}

\begin{abstract}
Ilona Du Bois, ${ }^{1,3, a, b}$ Annalisa Marsico, ${ }^{4,5, a}$ Wilhelm Bertrams, ${ }^{1,3, a}$ Michal R. Schweiger, ${ }^{6}$ Brian E. Caffrey, ${ }^{4}$ Alexandra Sittka-Stark, ${ }^{1,3, b}$ Martin Eberhardt, ${ }^{7}$ Julio Vera, ${ }^{7}$ Martin Vingron, ${ }^{4}$ and Bernd T. Schmeck ${ }^{1,2,3}$

${ }^{1}$ Institute for Lung Research/iLung, ${ }^{2}$ Department of Medicine, Pulmonary, and Critical Care Medicine, University Medical Center Marburg, Philipps-University, ${ }^{3}$ Universities of Giessen and arburg Lung Centre, German Center for Lung Research, ${ }^{4}$ Max Planck Institute for Molecular Genetics, ${ }^{5}$ Free University, Berlin, ${ }^{6}$ Functional Epigenomics, University of Cologne, and ${ }^{7}$ Laboratory of Systems Tumor Immunology, Department of Dermatology, Friedrich-Alexander University Erlangen-Nürnberg, University Hospital Erlangen, Germany
\end{abstract}

Background. Legionella pneumophila is a causative agent of severe pneumonia. Infection leads to a broad host cell response, as evident, for example, on the transcriptional level. Chromatin modifications, which control gene expression, play a central role in the transcriptional response to L. pneumophila.

Methods. We infected human-blood-derived macrophages (BDMs) with L. pneumophila and used chromatin immunoprecipitation followed by sequencing to screen for gene promoters with the activating histone 4 acetylation mark.

Results. We found the promoter of tumor necrosis factor $\alpha$-induced protein 2 (TNFAIP2) to be acetylated at histone H4. This factor has not been characterized in the pathology of L. pneumophila. TNFAIP2 messenger RNA and protein were upregulated in response to L. pneumophila infection of human-BDMs and human alveolar epithelial (A549) cells. We showed that L. pneumophilainduced TNFAIP2 expression is dependent on the NF- $\kappa \mathrm{B}$ transcription factor. Importantly, knock down of TNFAIP2 led to reduced intracellular replication of L. pneumophila Corby in A549 cells.

Conclusions. Taken together, genome-wide chromatin analysis of L. pneumophila-infected macrophages demonstrated induction of TNFAIP2, a NF- $\mathrm{KB}-$ dependent factor relevant for bacterial replication.

Keywords. A549; macrophage; Legionella pneumophila; TNFAIP2.

Legionella pneumophila, a gram-negative bacterium, was first identified in 1976 as the cause of an outbreak of severe pneumonia at a veterans meeting in Philadelphia; cases of severe pneumonia due to L. pneumophila have since been termed "Legionnaires' disease" [1]. In the human lung, the alveolar epithelium and macrophages constitute the main targets for microorganisms and play a key role in the initiation of the innate immune response and the defense against respiratory infection. Upon invasion, L. pneumophila forms a distinct Legionella-containing vacuole. In this niche, L. pneumophila replicates until initiation of host cell lysis [2].

Eukaryotic gene expression is controlled by epigenetic mechanisms, including histone modifications and chromatin

Received 21 January 2016; accepted 21 April 2016; published online 29 April 2016.

Presented in part: International Conference: Innate Immunity of the Lung-Improving Outcome in Pneumonia, Berlin, Germany, September 2012.

a. D. B., A. M., and W. B. are joint first authors.

bresent affiliations: AstraZeneca (I. D. B.) and Labor Berlin Services (A. S.-S.), Berlin, Germany.

Correspondence: B. T. Schmeck, Institute for Lung Research, Philipps University Marburg, Hans-Meerwein-Str. 2, 35041 Marburg, Germany (bernd.schmeck@uni-marburg.de).

The Journal of Infectious Diseases ${ }^{\circledR} \quad$ 2016;214:454-63

(C) The Author 2016. Published by Oxford University Press for the Infectious Diseases Society of America. All rights reserved. For permissions, e-mail journals.permissions@oup.com. DOI: 10.1093/infdis/jiw171 remodeling. Increasing evidence shows that bacteria manipulate the chromatin structure and transcriptional program of host cells through diverse mechanisms, influencing the host's innate immune response. Understanding the bacteria-induced deregulation of the host in the physiopathology of infectious diseases has important therapeutic implications and represents a novel research field to be explored [3-7]. Previous studies show that L. pneumophila modifies the epigenetic landscape by acetylation $[8,9]$ and can also influence host gene expression by methylation $[10,11]$. We aim to shed light on the cellular response to L. pneumophila infection by investigating genome-wide changes in the activating histone $\mathrm{H} 4$ acetylation mark (acH4), using chromatin immunoprecipitation followed by sequencing (ChIP-Seq) in L. pneumophila-infected human-blood-derived macrophages (BDMs). We found acH4 modification at the tumor necrosis factor $\alpha$ (TNF- $\alpha$ )-induced protein 2 (TNFAIP2) promoter. TNFAIP2, also called B94 or M-Sec, was first identified as a TNF- $\alpha$-inducible gene in endothelial cells [12], and it was described as a Plk-dependent phosphoprotein with activating properties on Toll-like receptor (TLR) signature genes [13]. We found TNFAIP2 to be subject to inflammatory regulators such as the $\mathrm{NF}-\kappa \mathrm{B}$ complex and to be highly expressed at the messenger RNA (mRNA) and protein levels during infection with 
L. pneumophila. Additionally, we show that TNFAIP2 significantly affects bacterial replication.

\section{METHODS}

\section{Bacterial Strains and Infection}

L. pneumophila Corby wild-type (WT) and Corby $\Delta d o t A \mathrm{mu}-$ tant were kindly provided by A. Flieger (Wernigerode, Germany). Corby $\Delta f l a A$ and Corby $\Delta f l a A / f l a A$ were kindly provided by K. Heuner (Berlin, Germany). All strains were routinely grown as described before [14]. Cells were infected with L. pneumophi$l a$ at indicated multiplicities of infection (MOIs).

\section{Cell Culture}

The type-II alveolar cell line A549 was obtained from American Type Culture Collection and cultured in Ham's F12 medium with L-glutamine and 10\% fetal calf serum (PAA Laboratories, Pasching, Austria) without antibiotics as described previously $[8,9,14]$.

Human monocytes were isolated from buffy coats obtained from the Center for Transfusion Medicine and Hemotherapy, University Hospital Giessen and Marburg, by Miltenyi magnetic-activated cell sorting CD14-positive selection according to the manufacturer's instructions. After culture for 6 days in Roswell Park Memorial Institute 1640 (1\% glutamine and 1\% human serum type $\mathrm{AB}$ ), experiments were performed. All donors gave informed written consent.

Chemical inhibitors for MEK1/2 (U0126), JNK (JNK II), p38 (SB202190), and IKK $\beta$ (IKK XIII; all from Calbiochem, Darmstadt, Germany) were preincubated on the cells for 120 minutes at $10 \mu \mathrm{M}$ before subsequent experiments.

\section{Mouse Bone Marrow-Derived Macrophages and Alveolar Macrophages}

Bone marrow-derived macrophages were prepared from femurs and tibiae of WT, TLR2 ${ }^{-1-}$, TLR2, $4^{-1-}$, and TRIF $^{-1-}$ mice (all on the C57BL/6N background, kindly provided by M. Schnare, Marburg, Germany) and cultivated as described previously [15]. From the WT mice, also alveolar macrophages were extracted by bronchoalveolar lavage. Alveolar macrophages were cultivated for 2 days in Roswell Park Memorial Institute plus $5 \%$ fetal calf serum and antibiotics. Upon removal of antibiotics, cells were infected with L. pneumophila at a MOI of 10 .

\section{Lactate Dehydrogenase (LDH) Release Assay}

LDH release in the supernatants of TNFAIP small interfering RNA (siRNA) - or control siRNA-transfected cells was analyzed 48 hours after infection with L. pneumophila, using the cytotoxicity detection kit (Roche Diagnostics) according to the supplier's protocol.

\section{RNA Interference}

A549 cells were transfected with the lipid-based transfection agent siPORT NeoFX (Life Technologies, Carlsbad, California) according to the manufacturer's protocol, with a $5 \mathrm{nM}$ final concentration of Silencer Select siRNA targeting TNFAIP2
mRNA per $8 \times 10^{4}$ cells. Nonspecific negative control siRNA was included as negative control.

\section{Quantitative Reverse-Transcription Polymerase Chain Reaction (RT-PCR)}

RNA was isolated using Isol-RNA Lysis Reagent (5 PRIME, Hamburg, Germany) and reverse transcribed with the HighCapacity cDNA Reverse Transcription Kit according to the manufacturer's protocol. Quantitative RT-PCR was performed on a ViiA 7 Real-Time PCR System, using Fast SYBR Green Master Mix (all from Life Technologies). The following primers were used: 5'-GACTTGGGCTCACAGATAAAGC-3' (sense) and 5'-CAGGCAGTTGTTGATGTTGG-3' (antisense), for TNFAIP2 human (NM_006291); 5'-CACCAGGTGGTCTC CTCTGACT-3' (sense) and 5'-GTGGTCGTTGAGGGCAATG3' (antisense), for glyceraldehyde 3-phosphate dehydrogenase human (NM_002046); 5'-AGCCTTGCTTGAAATGCGTA-3' (sense) and 5'-GAACCCCACTCCGCTACTTA-3' (antisense), for LINCRNA00677 human (HG506670.1); 5'-CTGGAGGC CATGTTCTTGTC-3' (sense) and 5'-GCGCTGAGACTCCAG CTCTA-3' (antisense), for TNFAIP2 murine (NM_009396.2); and 5'-GCGGCGGAAAATAGCCTTTG-3' (sense) and 5'-GA TCACACGTTCCACCTCATC-3' (antisense), for RPS18 murine (NM_011296.2).

\section{Western Blot}

Western blot was performed as described previously [16]. Immunodetection was performed with mouse anti-TNFAIP2

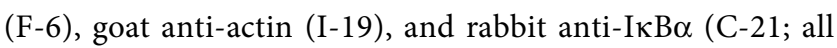
from Santa Cruz Biotechnology, Heidelberg, Germany) and visualized on a chemoluminescence imager (INTAS Science Imaging Instruments, Göttingen, Germany).

\section{Bacterial Replication Assay}

A549 cells were transfected with siRNA against TNFAIP2 and infected with L. pneumophila Corby WT for 1 hour. Subsequent bacterial replication was assessed as described previously [17].

\section{ChIP}

ChIP analyses were performed as described previously [14, 18, 19] with anti-RNA polymerase II (Pol II, N-20, Santa Cruz), antip65 (C-20), pan-anti-acetyl Histone H4 (06-598, Millipore, Darmstadt, Germany) and a rabbit control IgG antibody (ab46540, Abcam, Cambridge, United Kingdom) at $4^{\circ} \mathrm{C}$ overnight. The following TNFAIP2 (gene ID 7127) promoter-specific primers were used: 5'-GGCATGTCCCAGACCTTTC-3' (sense) and 5'-CACAGAGGGGGACTTTCACT-3' (antisense), for the proximal NF- $\kappa \mathrm{B}$ site; and 5'-ACAAAAGAGGGGAG GAGCAG-3' (sense) and 5'-CTCTGGAAAGGACCCAGA CA-3' (antisense), for the distal NF- $\kappa \mathrm{B}$ site.

For ChIP-Seq, the anti-acetyl-Histone H4 (06-598, Millipore) antibody and the MAGnify Chromatin Immunoprecipitation System were used according to the manufacturer's protocol (Life Technologies). 


\section{ChIP-Seq Data Analysis}

Library preparation was conducted with the Tru-Seq ChIP Sample Kit (Illumina, San Diego, California) according to the manufacturer's recommendations. Sequencing was done on an Illumina HiSeq2000 (Illumina) with a read length of 51 base pairs. Reads were mapped to the human genome (hg19) with the Bowtie Software (v1), allowing a maximum of 2 mismatches [20]. Genes were ranked according to the odds ratio (OR) of promoter acetylation in infected versus uninfected samples. For gene annotation, the ENSEMBL database v. 75 was used. Promoters were defined as \pm 1000 base pairs around the transcription start site. For each sample, the read counts per promoter were determined and corrected for the input (1), the enrichment of each sample over the input normalized by the median of the corrected read counts (1) was computed, and the OR of the infected samples over the control was determined (2), as follows:

$$
m_{\mathrm{ctrl} / \mathrm{inf}}=\operatorname{median}\left[\left(S_{\mathrm{ctrl} / \mathrm{inf}}+1\right) /\left(I_{\mathrm{ctrl} / \mathrm{inf}}+1\right)\right]
$$

and

$\left.\mathrm{OR}=\left[\left(S_{\mathrm{inf}}+1\right) / I_{\mathrm{inf}}+1\right)\right] /\left[\left(S_{\mathrm{ctrl}}+1\right) /\left(I_{\mathrm{ctrl}}+1\right)\left(m_{\mathrm{ctrl}} / m_{\mathrm{inf}}\right)\right]$,

where $m_{\mathrm{ctrl} / \text { inf }}$ denotes the median of the control or infected sample, $S_{\text {ctrl/inf }}$ denotes the read count of the control or infected sample, $I_{\mathrm{ctrl} / \text { inf }}$ denotes the read count of control or infected input, and 1 denotes the pseudo-count.

This formula corrects for unspecific high read count biases [21], which appear both in the input DNA and the precipitated samples. The ORs were log transformed to approximate a Gaussian distribution. If $\log _{(\mathrm{OR})} \geq 1$, the DNA was considered to show increased acetylation. If $\log _{(\mathrm{OR})} \leq-1$, decreased acetylation was assigned. For a detailed graphic representation of differentially acetylated regions, the UCSC Genome Browser was used [22]. GO terms and KEGG pathways for differentially acetylated genes was performed with the GSEABase R package [23]. Raw data are available under GSE79390.

\section{Transcriptome Analysis}

A549 cells were analyzed by MFT Services (Tübingen, Germany) on an Illumina HT12 BeadChIP MicroArray. Data analysis was performed using the lumi R package (Illumina microarray specific [24]). A variance-stabilizing transformation (lumi method) was used to background-corrected expression values, followed by quantile normalization between arrays. Differentially expressed genes were identified on the basis of the moderated $t$ test (limma R package [25]). Raw data are available under GSE80214.

\section{Transcription Factor-Binding Site (TFBS) Prediction}

The genomic sequence corresponding to the region 5000 base pairs upstream and 1000 base pairs downstream of the transcription start site of the TNFAIP2 gene was extracted from ENSEMBL, version 75. This sequence was scanned using Transfac positional weight matrices [26], employing the program matrix- scan (RSAT suit [27]) to identify TFBSs corresponding to proximal or distal regulatory elements. Transcription factor-binding site enrichment at core promoters of differentially acetylated genes was performed using Transfind [28]. Based on known transcription factor weight matrix representations, Transfind identifies enriched factors for a set of genes on the basis of computed binding affinities of each factor for the promoters in the gene set. We restricted our analysis to core promoters (300-base pair regions upstream and 200-base pair regions downstream of the transcription start sites) of differentially acetylated genes with a cutoff of 1.0 on the logarithmic fold change.

\section{Ethical Statement}

Animals were handled according to European Union Council Directive 86/609/EEC for the protection of animals. The performed protocols were approved by the responsible animal ethics committee at Philipps-University Marburg ( permit numbers EX-22-2013, EX-9-2014, and EX-3-2016).

\section{Statistical Analyses}

Data are presented as means \pm standard errors of the mean of at least 3 independent experiments. Effects were statistically evaluated using the Student $t$ test. $P$ values of $<.05$ were considered statistically significant.

\section{RESULTS}

Genome-wide Mapping of $\mathrm{H} 4$ Acetylation in L. pneumophila-Infected Blood-Derived Macrophages Identifies the TNFAIP2 Promoter

ChIP-Seq analysis of BDMs infected with L. pneumophila Corby for 1 hour identified 3715 genes that showed increased (494 genes [13\%]) or decreased (3221 genes [87\%]) H4 acetylation of at least 2-fold, compared with uninfected BDMs (Figure 1, Supplementary Table 1, and Supplementary Table 2). While $60 \%$ of the differentially acetylated genes (2246 of 3715) were protein coding, 11\% (396 of 3715) encoded long intergenic noncoding RNAs (lincRNAs), 8\% (281 of 3715) encoded small noncoding RNAs (microRNAs, miscellaneous RNAs, small nuclear RNAs, and small nucleolar RNAs), and 21\% (792 of 3715) encoded genes of other categories, such as pseudogenes and antisense transcripts (Figure 1A). A gene set enrichment analysis returned sets of acetylated genes significantly enriched in cytokine activity, cytokine receptor binding, TNF receptor binding, immune response, and response to bacterium, according to the Gene Ontology Database (Supplementary Figure 1). The same analysis performed on KEGG pathways returned sets of acetylated genes that are significantly enriched in pathways such as cytokine-cytokine receptor interaction and TLR signaling pathway (among the top 10; Supplementary Figure 1). Investigation of deacetylated genes by KEGG pointed to enrichment in the gene encoding lysosome (Supplementary Figure 1). Important regulated genes with relevance for inflammation and also chemotactic recruitment of further immune cells include those encoding interleukin $1 \beta$ (IL-1 $\beta$ ) and TNF (Supplementary 


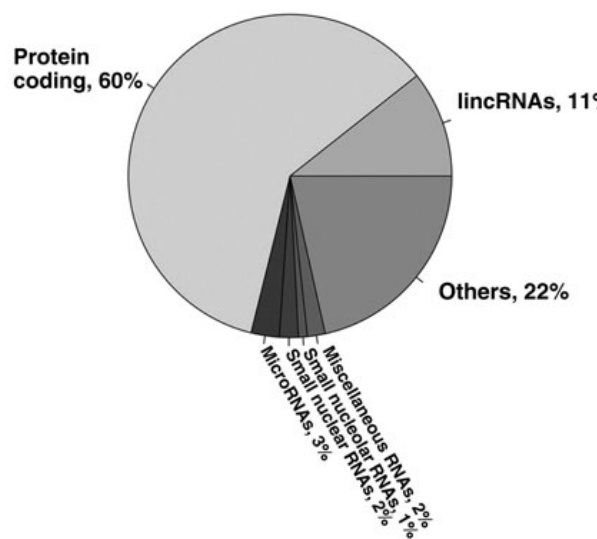

C

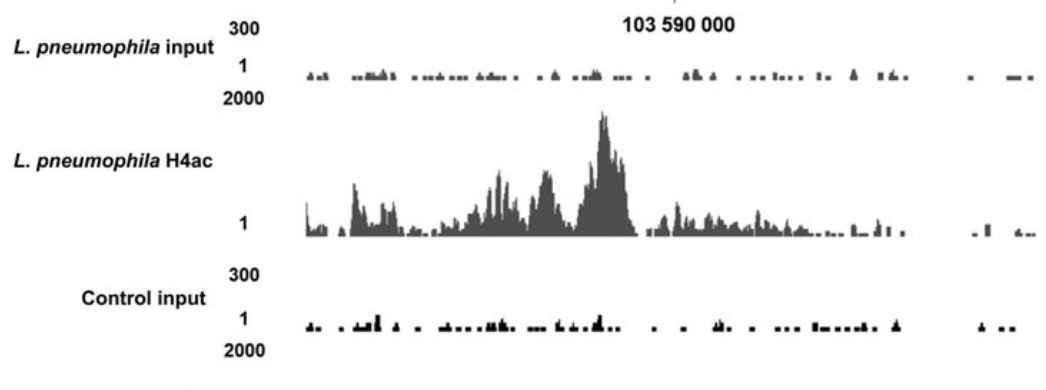

Control H4ac

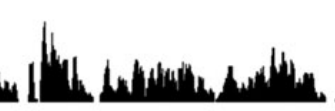

Positive strand

Negative strand
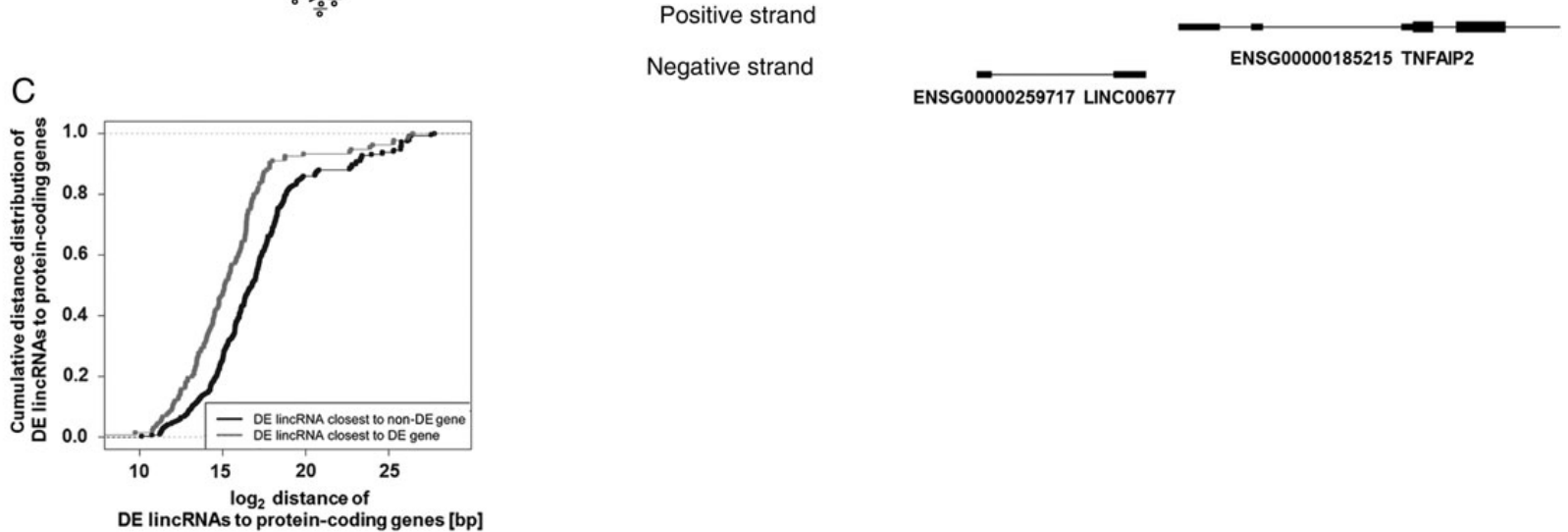

ENSG00000259717 LINC00677

Figure 1. Chromatin immunoprecipitation followed by sequencing analysis reveals high acetylation of histone $\mathrm{H} 4$ (acH4) at the tumor necrosis factor $\alpha$-induced protein 2 (TNFAIPZ) promoter. Blood-derived macrophages (BDMs) were infected with Legionella pneumophila Corby for 1 hour at a multiplicity of infection of 10. acH4-immunoprecipitated fractions were sequenced on an Illumina HiSeq2000. A, Of all genes that showed a change in acH4 acetylation upon infection, 60\% were protein coding, while $19 \%$ were long and short noncoding RNAs. B, The TNFAIP2 promoter, located on chromosome 14, showed a strong increase of histone $\mathrm{H} 4$ acetylation as indicated by the read coverage in the UCSC Genome Browser (reference genome, hg19). While the TNFAIP2 gene is encoded on the positive DNA strand, the long intergenic noncoding RNA (lincRNA) LINC00677 was found on the negative strand. The read coverage for each library was normalized to $109 / n$, where $n$ equals the sum of all reads per library. $C$, Genes that are deregulated (DE) in acH4 after infection (gray curve) are significantly closer (median distance, $44 \mathrm{~kb}$ ) to deregulated lincRNAs $\left(P=2.53 \times 10^{-8}\right.$, by the Wilcoxon sum rank test) than nondifferentially regulated genes (black curve; median distance, $144 \mathrm{~kb}$ ) with regard to the cumulative distribution of the distances of deregulated lincRNAs to the closest protein-coding gene.

Table 6). Transcription factor (TF) binding analyses of genes with a logarithmic fold change of at least 1.0 returned TFs enriched for the core promoters of differentially acetylated genes (Supplementary Tables 3 and 4). Among them we found some well-known factors that regulate the immune response, such as SP1, NFKB1, and KLF4. Ingenuity Pathway Analysis of the acetylated genes revealed that they were subject to proinflammatory upstream regulators, among them TNF (activation $z$ score, 6.63; $P=1.56 \times 10^{-20}$ for overlap), the NF- $\mathrm{BB}$ complex (5.77, $\left.2.34 \times 10^{-20}\right)$, and IL-1 $\beta\left(5.52,4.31 \times 10^{-20}\right.$; data not shown). Conversely, deacetylated genes were targets, among others, of IFNA2 $\left(-6.066 ; 1.76 \times 10^{-11}\right)$, and the IFN $\beta$ group $(-4.631$; $5.37 \times 10^{-06}$; data not shown), which is in line with a reported inhibition of the type I interferon response by the L. pneumophila factor SdhA [29]. The TNFAIP2 promoter was strongly acetylated as compared to that for uninfected control macrophages (Figure $1 B$ ), and TNFAIP2 is part of both the TNF network and the NF- $\mathrm{\kappa B}$ network, suggesting a role of TNFAIP2 in the immune response to L. pneumophila. Furthermore, we found the lincRNA 00677 was encoded on the negative strand, and we hypothesized it to be controlled by the TNFAIP2 promoter. Accordingly, we showed that lincRNA 00677 is upregulated shortly after L. pneumophila infection (Supplementary Figure 2) and precedes TNFAIP2 induction, which we could not detect at these early time points (data not shown). A global analysis of lincRNAs showed that acH4-modified lincRNA genes are spatially closer to acH4-modified protein-coding genes than to unmodified protein-coding genes (Figure 1C).

\section{L. pneumophila Stimulates Expression of TNFAIP2 in Human Myeloid and Alveolar Epithelial Cells at the mRNA and Protein Levels and in Murine Primary Alveolar Macrophages at the mRNA Level}

It is known that TNFAIP2 is rapidly induced in TNF- $\alpha$-treated epithelial cells [12].L. pneumophila induced TNFAIP2 mRNA expression in a time- and MOI-dependent manner in A549 cells 

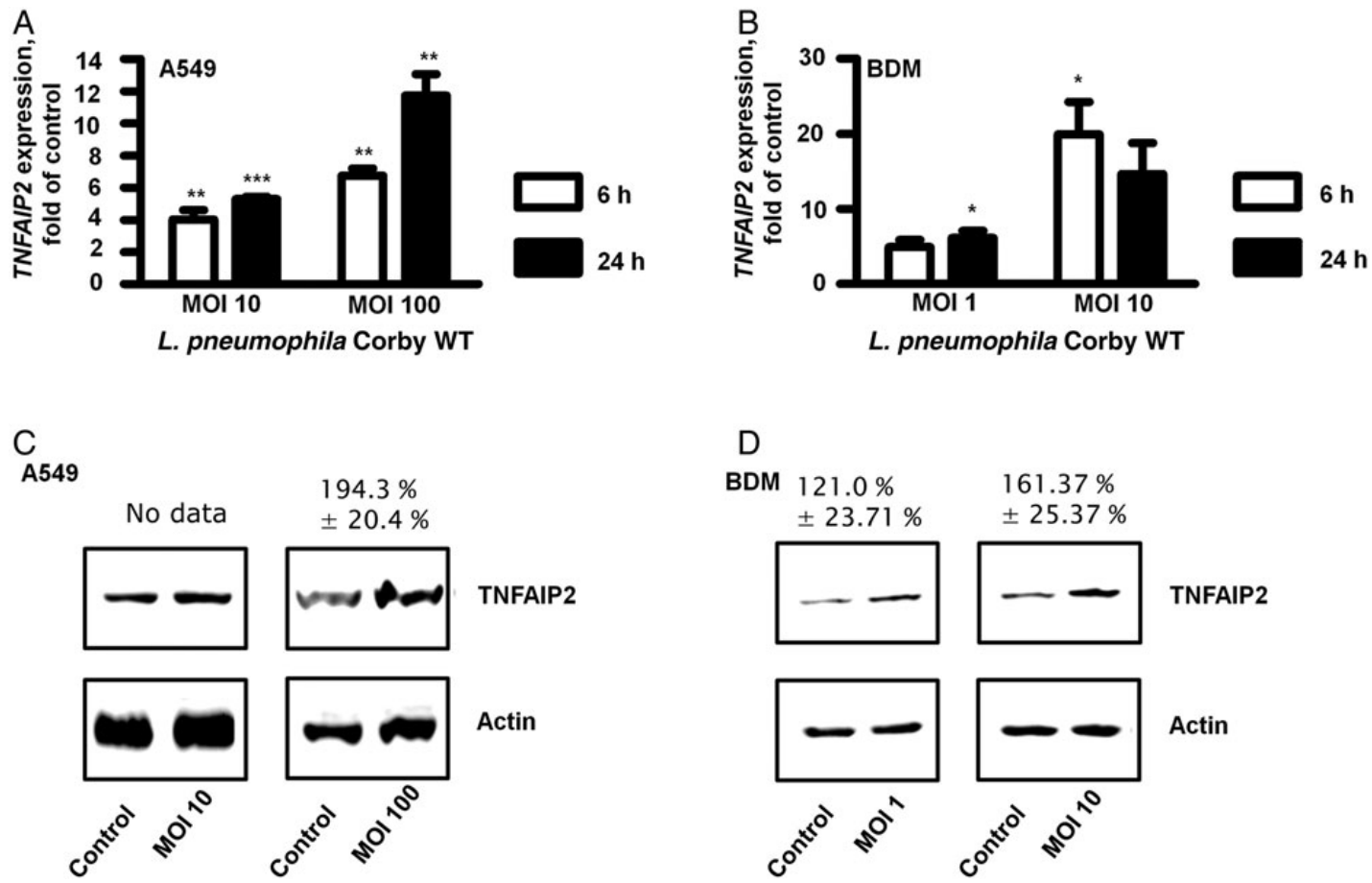

Figure 2. The gene encoding tumor necrosis factor $\alpha$-induced protein 2 (TNFAIP2) is induced upon Legionella pneumophila infection in lung epithelial cells and myeloid cells. A549 cells $(A$ and $B)$ and blood-derived macrophages (BDMs; $C$ and $D)$ were infected with $L$. pneumophila Corby at multiplicities of infection of 10 and $100(A$ and $B$, respectively) and 1 and $10(C$ and $D$, respectively) for 6 hours and 24 hours $(A$ and $C)$ or 6 hours $(B$ and $D)$. TNFAIP2 levels were detected by quantitative polymerase chain reaction (qPCR; $A$ and $C$ ) and Western blot $(B$ and $D)$. One representative blot of 3 is shown. An increase in protein expression is presented as a percentage of the control and was calculated from densitometric analysis of 3 independent experiments. qPCR data $(A, C, E$, and $F)$ are normalized against glyceraldehyde 3-phosphate dehydrogenase and shown as means \pm standard errors of the mean of at least 3 independent experiments. ${ }^{*} P<.05,{ }^{* *} P<.01$, and ${ }^{* *} P<.001$ vs untreated control. Abbreviations: M0l, multiplicity of infection; WT, wild type.

(Figure 2A) and BDMs (Figure 2C) 6 and 24 hours after infection. This induction could be enhanced by raising the bacterial load (A549: MOI range, 10-100; BDM, MOI range, 1-10). At the protein level, the mean TNFAIP2 level ( \pm standard errors of the mean [SEM]) in A549 cells 6 hours after infection was 194.3\% $\pm 20.4 \%$ of the basal level (MOI, 100; Figure 2B), and the mean BMD level $( \pm$ SEM) 6 hours after infection was $161.37 \% \pm 25.37 \%$ of the basal level (MOI, 10; Figure 2D), as calculated from 3 independent experiments. To corroborate these findings in a more physiologic setting, we analyzed primary murine alveolar macrophages and also found upregulation of TNFAIP2 after infection with L. pneumophila $\Delta$ FlaA (Supplementary Figure 3).

\section{L. pneumophila-Induced Expression of TNFAIP2 Depends on Flagellin and on the Legionella Type-IVB Dot/lcm Secretion System}

We stimulated A549 cells with recombinant flagellin or infected the cells with the immotile L. pneumophila Corby $\Delta$ flaA or the type IVB-deficient $\triangle \operatorname{dot} A$ mutants to identify crucial bacterial components for TNFAIP2 induction (Figure 3A). Six hours after infection, we observed that TNFAIP2 expression is dependent on both flagellin and an intact type-IVB Dot/Icm secretion system, as the $\triangle$ flaA and $\triangle \operatorname{dot} A$ mutants induced less TNFAIP2 transcription, and flagellin alone only moderately induced TNFAIP2. The flagellin-complemented $\triangle$ flaA mutant $(\Delta$ flaA/
flaA) performed as the WT strain. At 24 hours after infection, TNFAIP2 expression seems to be solely dependent on a functional type-IVB Dot/Icm secretion system, since flagellindeficient $L$. pneumophila were able to induce TNFAIP2 like the WT strain. Induction of TNFAIP2 is achieved by TLR2 and TLR 5 signaling at 6 hours, as illustrated by the comparatively weak induction of TNFAIP2 in TLR2 $2^{-1-}$ and TLR $2,4^{-1-}$ cells upon infection with L. pneumophila $\triangle$ flaA, as opposed to findings for WT cells and WT L. pneumophila. TRIF ${ }^{-1-}$ cells showed no differential induction of TNFAIP2 under any tested condition (Figure 3B).

\section{L. pneumophila-Induced Expression of TNFAIP2 Depends on NF-кB Activation}

L. pneumophila activates a plethora of signaling pathways, including the MAPK pathways and the NF- $\kappa B$ pathway, upon infection of lung epithelial cells [30,31]. In BDMs, we found a rapid degradation of $\mathrm{I} \kappa \mathrm{B} \alpha$ upon infection (Supplementary Figure 4). To determine the signaling pathways involved in L. pneumophila-induced activation of TNFAIP2, we incubated A549 cells with the MAPK inhibitors U0126 (MEK1), JNK II (JNK), SB202190 (p38), and the NF- $\mathrm{BB}$ inhibitor IKK XIII 120 minutes before infection. TNFAIP2 mRNA (Figure $4 A$ ) and protein (Figure $4 B$ ) expression was solely influenced by 


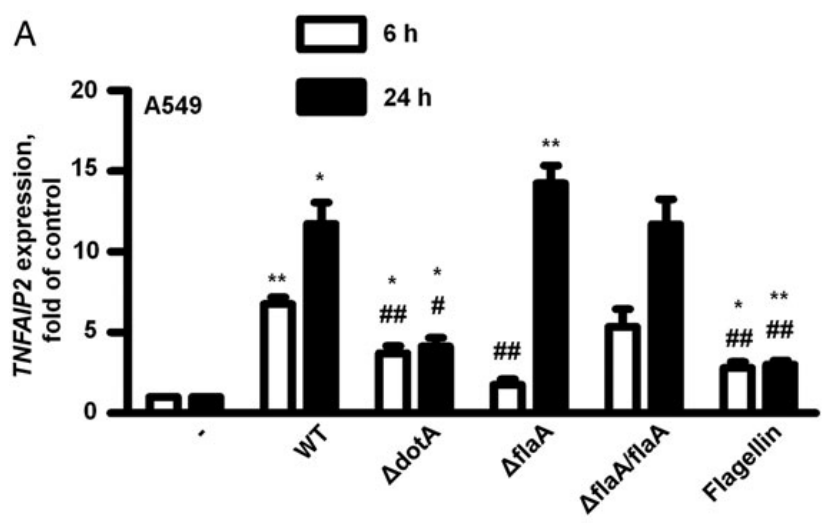

B
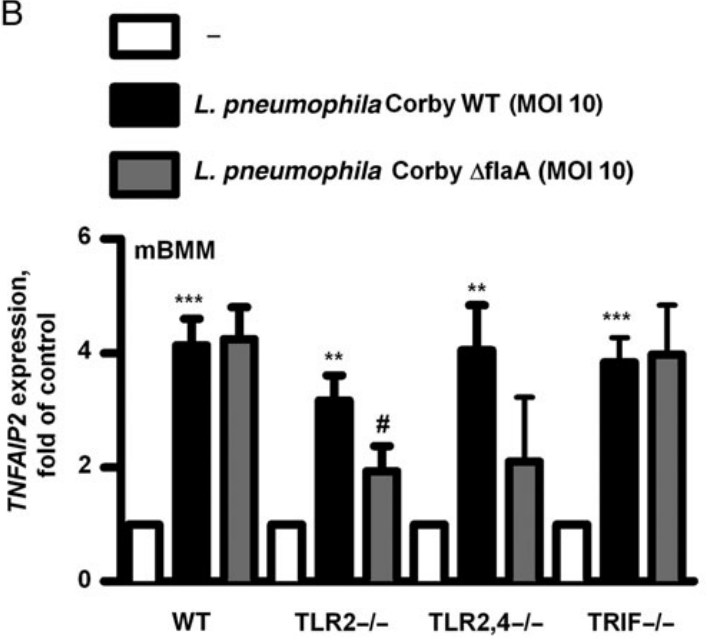

Figure 3. Tumor necrosis factor $\alpha$-induced protein 2 (TNFAIPZ) induction requires flagellin and the type-IVB Dot/lcm secretion system. A, A549 cells were infected with Legionella pneumophila mutants at a multiplicity of infection (MOI) of 100 or stimulated with flagellin $(10 \mathrm{ng} / \mathrm{mL})$ for 6 hours and 24 hours. $B$, Murine bone marrow macrophages (mBMMs) from wild-type (WT), TLR2 ${ }^{-1-}, \mathrm{TLR}_{2}, 4^{-/-}$, and $\mathrm{TRIF}^{-/}$mice were infected with L. pneumophila WT at a multiplicity of infection (MOI) of 10 for 6 hours. TNFAIP2 levels were detected by quantitative polymerase chain reaction and normalized against glyceraldehyde 3-phosphate dehydrogenase. Data are shown as means \pm standard errors of the mean of at least 3 independent experiments. ${ }^{*} P<.05$, ${ }^{* *} P<.01$, and ${ }^{* * *} P<.001$ vs untreated control; ${ }^{\#} P<.05,{ }^{\# \#} P<.01$, and ${ }^{\# \# \#} P<.001$ vs infection with $L$. pneumophila Corby WT.

NF- $\kappa \mathrm{B}$ inhibition. Transcription factor binding site analysis identified 2 NF- $\kappa \mathrm{B}$-binding sites, one 419-435 base pairs upstream (first site, proximal) and one 3994-4002 base pairs upstream (second site, distal) of the transcription start site. Since our data indicate a role for NF- $\mathrm{KB}$ in TNFAIP2 expression, we used ChIP to assess the interaction of the NF- $\mathrm{B}$ subunit $\mathrm{p} 65$ and RNA polymerase II with the TNFAIP2 promoter region. In L. pneumophila-infected A549 cells, we observed a timedependent binding of NF- $\kappa$ B subunit p65 exclusively at the distal (second) site (Figure 4C). Binding of p65 was accompanied by the recruitment of RNA polymerase II, which could be observed at the proximal (first) site, indicating transcription (Figure 4D).
TNFAIP2 Knock Down Reduced Intracellular L. pneumophila Replication but Did Not Significantly Change the Overall Gene Expression Pattern

The upregulation of TNFAIP2 upon infection with L. pneumophila suggests a role in host-pathogen interaction. We transfected A549 cells with TNFAIP2-specific or control siRNA. Knock down was confirmed by quantitative PCR (Figure $5 A$ ) and Western blot (Figure 5B). interleukin 8 (IL-8) expression was not altered in infected cells after knock down of TNFAIP2 (Figure $5 C$ ), but we found that intracellular growth of L. pneumophila was reduced by $50 \%$ in TNFAIP2 siRNA-transfected cells after 48 hours (Figure 5D). This reduction was not due to increased cell death in the TNFAIP2 siRNA-transfected cells, as demonstrated by the LDH assay (Supplementary Figure 5). These data suggest that TNFAIP2 is relevant for intracellular growth of L. pneumophila in A549 cells, while it is not necessary for general proinflammatory activation as shown by unaltered IL-8 release. To characterize this in more detail, we performed mRNA microarrays from infected A549 cells transfected with control or TNFAIP2-specific siRNA. We compared 3 experimental conditions (infected/scramble and infected/TNFAIP2si vs uninfected/untransfected) and found a distinctly proinflammatory response characterized by genes that were induced irrespective of the knock down (Supplementary Table 5). These genes mapped primarily to the pathways immune system, innate immune system, cytokine signaling in immune system, and cellular responses to stress. Furthermore, highly significant enrichment could be observed for diseases associated with the TLR signaling cascade and diseases of the immune system (Figure 6). We did not find an impact of TNFAIP2 knock down on the transcriptome, so we investigated in silico a possible function at the protein level. TNFAIP2 is reported to interact with proteins of the exocyst complex, according to the STRING database [32]. While some of these interactions are based on observations in humans, others rely on homologues found in yeast (Supplementary Figure 6).

\section{DISCUSSION}

Infectious agents such as L. pneumophila cause a shift in the epigenetic landscape. A prominent activating epigenetic modification is acH4. To obtain a comprehensive genome-wide acH4 pattern, we performed ChIP-Seq in human blood-derived macrophages infected with L. pneumophila. Our enrichment analysis revealed that genes with induced acetylation upon infection are enriched in immune response pathways. Among the genes with increased acH4 acetylation, we found numerous candidates that contribute to the host response against Legionella. IL-1B and TNF, for example, are potent proinflammatory cytokines that positively influence neutrophil chemotaxis. In an in vivo Legionella infection, these factors might contribute to the recruitment of neutrophils. We can also show that proteincoding genes and lincRNA genes tend to be spatially closer to 


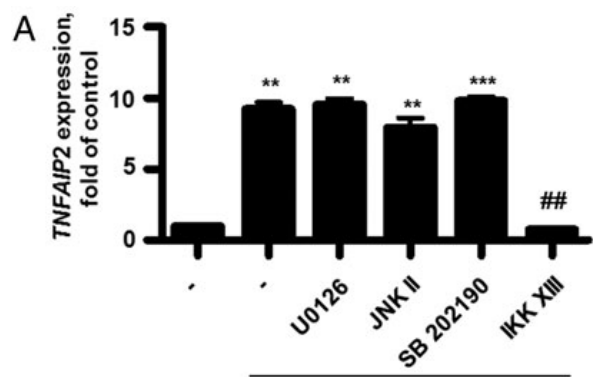

L. pneumophila Corby WT (MOI 100)

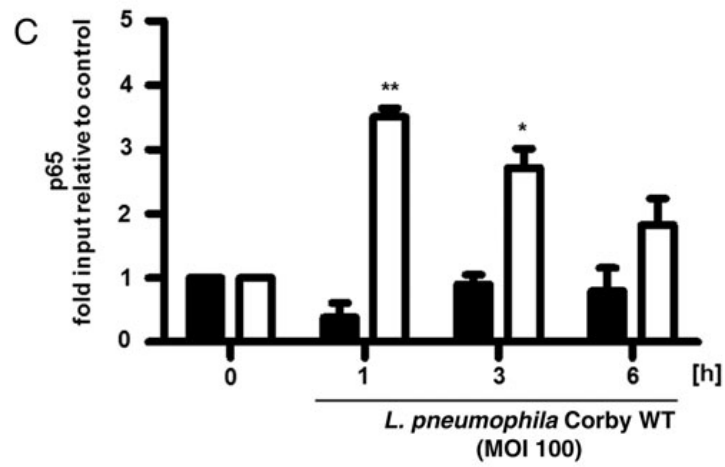

First NF-KB site
B
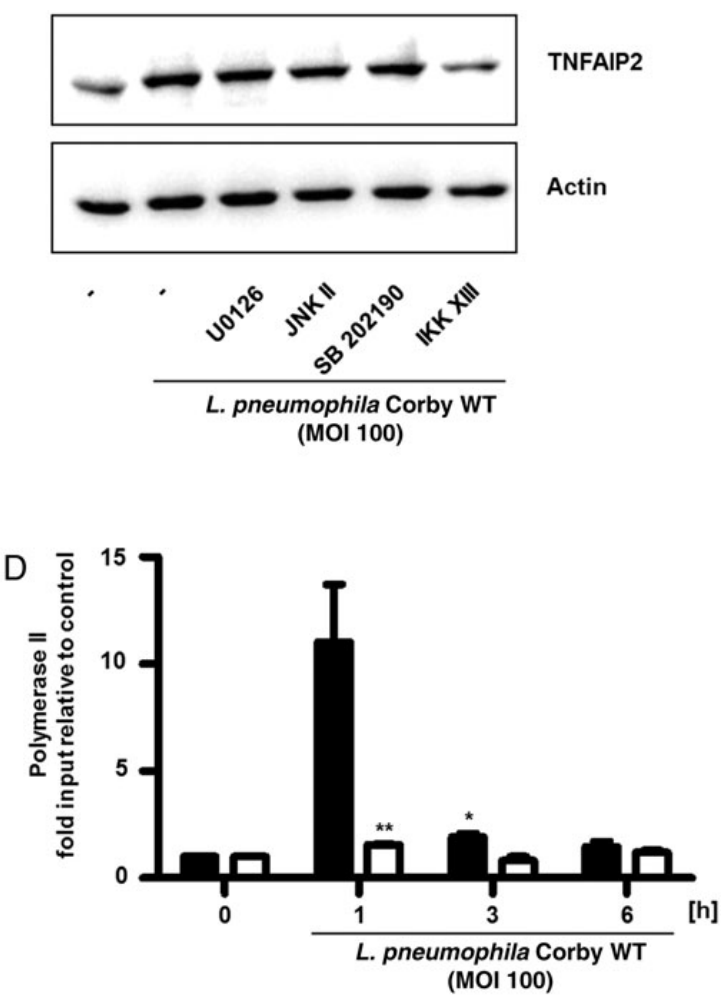

First NF-KB site
Second NF-KB site

Figure 4. Legionella pneumophila-induced tumor necrosis factor $\alpha$-induced protein 2 (TNFAIP2) expression depends on NF- $\mathrm{KB}$ recruitment. A549 cells were incubated for 120 minutes with the MAP kinase inhibitors U0126 (ERK1/2), JNK II (JNK), and SB2012190 (p38) and the NF-kB inhibitor IKK XIII (10 $\mu \mathrm{M}$ each) and then infected with L. pneumophila Corby at a multiplicity of infection (MOI) of 100 for 6 hours. $A$ and $B$, TNFAIP2 messenger RNA levels were determined by quantitative polymerase chain reaction (qPCR; $A$ ), and TNFAIP2 expression was detected by Western blot (B). One representative Western blot experiment of 3 is shown (B). $C$ and $D$, A549 cells were infected with L. pneumophila Corby at a $\mathrm{MOI}$ of 100 for the indicated time points ( $C$ and $D)$. Chromatin immunoprecipitation was performed against the NF-kB subunit p65 and po-

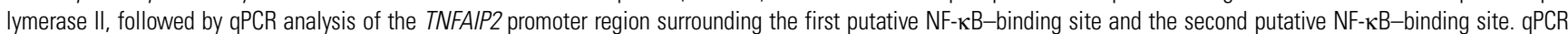
data are shown as means \pm standard errors of the mean of at least 3 independent experiments. ${ }^{*} P<.05,{ }^{*} P<.01$, and ${ }^{* * *} P<.001$ vs untreated control; ${ }^{\#} P<.05$, ${ }^{\# \#} P<.01$, and ${ }^{\# \# \# P} P$.001 vs infection without inhibitor. Abbreviation: WT, wild-type.

each other in case they are both acH4-modified upon infection, indicating that they are coregulated or that lincRNAs might regulate protein-coding genes in cis. Among others, we could identify an increased acetylation pattern at the promoter of the TNFAIP2 gene. Promoter region analysis revealed its bidirectional nature, and we show that the lincRNA 00677, which is encoded on the
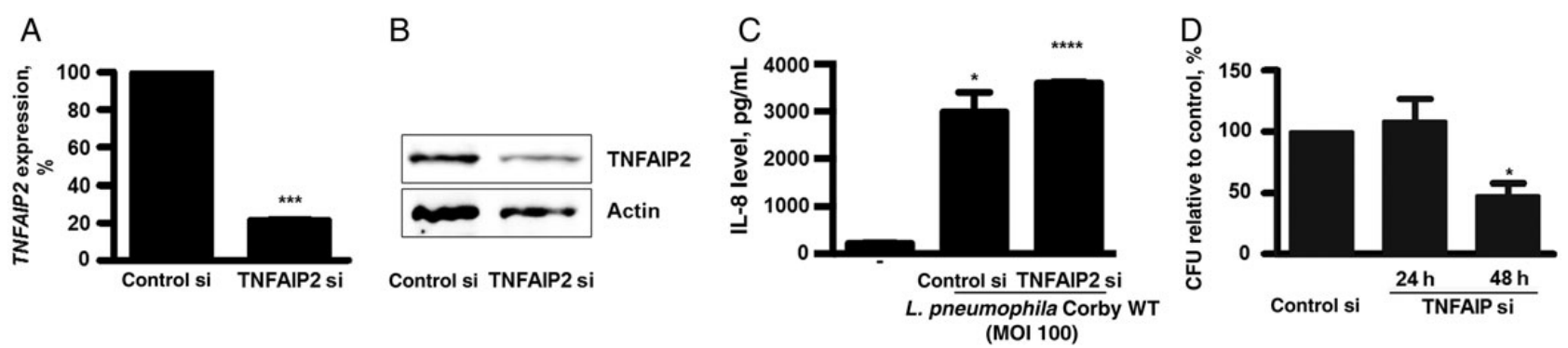

Figure 5. Knock down of tumor necrosis factor $\alpha$-induced protein 2 (TNFAIP2) in A549 cells leads to reduced intracellular replication of Legionella pneumophila. A549 cells were subjected to dsRNA transfection with control or TNFAIP2-targeting small interfering RNA (siRNA) for 24 hours. $A$ and $B$, Knock down of TNFAIP2 was verified by quantitative polymerase chain reaction $(A)$ and Western blot $(B)$. One representative Western blot experiment of 3 is shown $(B)$. $C$ and $D$, Transfected cells were infected with L. pneumophila Corby at a multiplicity of infection (MOI) of 100 for 6 hours $(C)$ or 2 hours (D). Interleukin 8 (IL-8) release was determined by enzyme-linked immunosorbent assay $(C)$. Bacterial replication was monitored over 48 hours by determining colony-forming units (CFU) per well (D). Data are shown as means \pm standard errors of the mean of at least $2(C)$ or $3(A$ and $D)$ independent experiments. ${ }^{*} P<.05,{ }^{* *} P<.01,{ }^{* * *} P<.001$, and ${ }^{* * *} P<.0001$ vs untreated control. Abbreviation: WT, wild-type. 


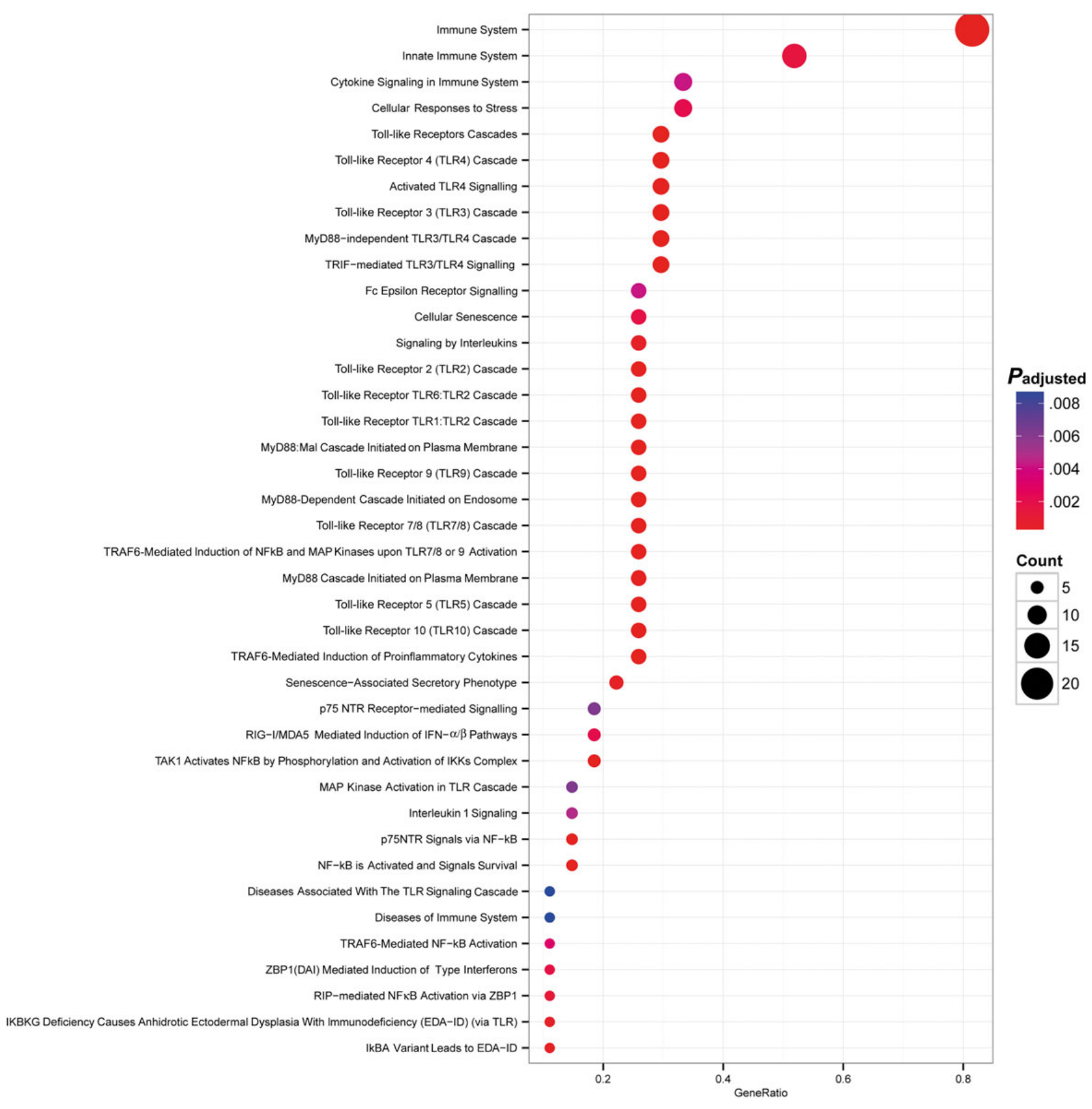

Figure 6. Pathway overrepresentation analysis of gene sets, using the ReactomePA bioconductor R package. We compared the gene expression patterns in the infected/ scramble and infected/ tumor necrosis factor $\alpha$-induced protein 2 (TNFAIP2)si versus the uninfected/untransfected condition, respectively, and found common genes in both comparisons that point to a pro-inflammatory activation after Legionella pneumophila infection. Dot sizes correspond to the number of genes in each pathway. Dot colors represents the $P$ values corresponding to each pathway. The $x$-axis shows the ratio of the number of genes in the set that are in each pathway. Some pathways include overlapping sets of genes.

negative strand, is induced early after infection and precedes TNFAIP2 expression. Whether this lincRNA is involved in initiation of TNFAIP2 expression remains to be elucidated. We furthermore found $2 \mathrm{NF}-\mathrm{\kappa B}-$ binding sites at this promoter. While the proximal site accommodates RNA polymerase, the distal site recruits the NF- $\mathrm{KB}$ p 65 subunit, as has been recently shown [33]. This observation supports our hypothesis of an involvement of TNFAIP2 in the response to bacterial infection.

Induction of TNFAIP2 relied on flagellin, the type IVB Dot/ Icm secretion system, and NF- $\mathrm{KB}$, as its inhibition completely abrogated the TNFAIP2 response, as has been shown elsewhere [33]. This mechanism puts TNFAIP2 into a central proinflammatory signaling hub. We observed a significant decrease in 
bacterial load in infected A549 cells with TNFAIP2 knock down, but no change in the proinflammatory response against $L$. pneumophila was evident. IL-8 mRNA, a common indicator of proinflammatory activation upon infection [8], remained unaltered by TNFAIP2 knock down, as did major other proinflammatory genes that map to important immune pathways. A comparison of our data with the GSE61535 macrophage data set [34] yielded a good correlation of the upregulated genes, as evidenced by a Pearson product moment correlation test $(\mathrm{rho}=0.358$; $P=8.6 \times 10^{-5}$ ). Upregulated genes included important immune factors, such as IL-8, CXCL2, CXCL1, and PTGS2. As no impact on the transcriptome by TNFAIP2 knock down under infectious conditions was evident, we focused on possible protein interactions. In a relevant study, TNFAIP2 was shown to coprecipitate with CDC5L in a protein-binding assay. In turn, CDC5L binds Sec5, which is part of the hetero-octameric exocyst complex [35]. Another subunit of the exocyst complex, Exo84, has been reported to be necessary for amino acid starvation-induced autophagy [36]. This indirect functional association between TNFAIP2 and the exocyst is corroborated by several sources from the STRING database. TNFAIP2 is reported to have a high-confidence direct physical interaction with Sec5 (EXOC2), supported by experimental evidence of homologous proteins in yeast. In addition, several direct high-confidence interactions of TNFAIP2 with other components of the exocyst complex are reported by STRING (EXOC1, EXOC4, EXOC6, EXOC7, EXOC8, and EXOC6B), as well as proteins involved in intracellular vesicular trafficking (STXBP1, STXBP2, and STXBP3). This hints toward involvement of vesicular trafficking in the observed impaired L. pneumophila growth in TNFAIP2-knock down cells.

Altogether, we provide evidence that $L$. pneumophila infection of human primary macrophages leads to activation of TNFAIP2, whose expression at early stages of infection mainly depends on NF- $\kappa$ B. The significant achievement of this work is the identification of a new gene in the L. pneumophila-induced host gene expression landscape with an active role in bacterial replication. Our future analyses will focus on the identified noncoding RNAs of yet unknown function, such as the linc00677 described herein, to show how they contribute to the gene expression changes of nearby genes and in particular those genes involved in the host response to L. pneumophila.

\section{Supplementary Data}

Supplementary materials are available at http://jid.oxfordjournals.org. Consisting of data provided by the author to benefit the reader, the posted materials are not copyedited and are the sole responsibility of the author, so questions or comments should be addressed to the author.

\section{Notes}

Acknowledgments. We thank Franziska Seifert (Charité, University Medicine Berlin, Germany), Kerstin Hoffmann (Philipps University Marburg, Germany), and Nadine Siebert (Philipps University Marburg), for excellent technical assistance; and Florian Strassheimer, for the internship he dedicated to this work.
Financial support. The work was supported by the Volkswagenstiftung (Lichtenberg program) to M. R. S., Deutsche Forschungsgemeinschaft (TR84) to B. T. S. and M. V., Bundesministerium fuer Bildung und Forschung (e:bio (0316175A, 0316175B), e:Med (01X1304E, 01ZX1304F)) to B. T. S. and J. V., Progress (FKZ 01KI1010K) to B. T. S. and Hessisches Ministerium fuer Wissenschaft und Kunst (LOEWE UGMLC and LOEWE Medical RNomics) to B. T. S.

Potential conflict of interest. All authors: No reported conflicts. All authors have submitted the ICMJE Form for Disclosure of Potential Conflicts of Interest. Conflicts that the editors consider relevant to the content of the manuscript have been disclosed.

\section{References}

1. Fraser DW, Tsai TR, Orenstein W, et al. Legionnaires' disease: description of an epidemic of pneumonia. N Engl J Med 1977; 297:1189-97.

2. Isaac DT, Isberg R. Master manipulators: an update on Legionella pneumophila Icm/Dot translocated substrates and their host targets. Future Microbiol 2014; 9:343-59.

3. Bierne H, Hamon M, Cossart P. Epigenetics and bacterial infections. Cold Spring Harb Perspect Med 2012; 2:a010272.

4. Paschos K, Allday MJ. Epigenetic reprogramming of host genes in viral and microbial pathogenesis. Trends Microbiol 2010; 18:439-47.

5. Minarovits J. Microbe-induced epigenetic alterations in host cells: the coming era of patho-epigenetics of microbial infections. A review. Acta Microbiol Immunol Hung 2009; 56:1-19.

6. Hamon MA, Cossart P. Histone modifications and chromatin remodeling during bacterial infections. Cell Host Micro 2008; 4:100-9.

7. Rolando M, Buchrieser C. Legionella pneumophila type IV effectors hijack the transcription and translation machinery of the host cell. Trends Cell Biol 2014; 24:771-8.

8. Lorenz J, Zahlten J, Pollok I, et al. Legionella pneumophila-induced IkappaBzetadependent expression of interleukin-6 in lung epithelium. Eur Respir J Suppl 2011; 37:648-57.

9. Schmeck B, Lorenz J, N'Guessan PD, et al. Histone acetylation and flagellin are essential for Legionella pneumophila-induced cytokine expression. J Immunol 2008; 181:940-7.

10. Li T, Lu Q, Wang G, et al. SET-domain bacterial effectors target heterochromatin protein 1 to activate host rDNA transcription. EMBO Rep 2013; 14:733-40.

11. Rolando M, Sanulli S, Rusniok C, et al. Legionella pneumophila effector RomA uniquely modifies host chromatin to repress gene expression and promote intracellular bacterial replication. Cell Host Microbe 2013; 13:395-405.

12. Sarma V, Wolf FW, Marks RM, Shows TB, Dixit VM. Cloning of a novel tumor necrosis factor-alpha-inducible primary response gene that is differentially expressed in development and capillary tube-like formation in vitro. J Immunol 1992; 148:3302-12.

13. Chevrier N, Mertins P, Artyomov MN, et al. Systematic discovery of TLR signaling components delineates viral-sensing circuits. Cell 2011; 147:853-67.

14. Witzenrath M, Gutbier B, Owen JS, et al. Role of platelet-activating factor in pneumolysin-induced acute lung injury. Crit Care Med 2007; 35:1756-62.

15. Sester DP, Trieu A, Brion K, et al. LPS regulates a set of genes in primary murine macrophages by antagonising CSF-1 action. Immunobiology 2005; 210:97-107.

16. Krull M, Kramp J, Petrov T, et al. Differences in cell activation by Chlamydophila pneumoniae and Chlamydia trachomatis infection in human endothelial cells. Infect Immun 2004; 72:6615-21.

17. Lippmann J, Muller HC, Naujoks J, et al. Dissection of a type I interferon pathway in controlling bacterial intracellular infection in mice. Cell Microbiol 2011; $13: 1668-82$.

18. Schmeck B, Beermann W, van Laak V, et al. Intracellular bacteria differentially regulated endothelial cytokine release by MAPK-dependent histone modification. J Immunol 2005; 175:2843-50.

19. Schmeck B, Gross R, N'Guessan PD, et al. Streptococcus pneumoniae-induced caspase 6-dependent apoptosis in lung epithelium. Infect Immun 2004; 72:4940-7.

20. Langmead B, Trapnell C, Pop M, Salzberg SL. Ultrafast and memory-efficient alignment of short DNA sequences to the human genome. Genome Biol 2009; 10:R25.

21. Perner J, Lasserre J, Kinkley S, Vingron M, Chung HR. Inference of interactions between chromatin modifiers and histone modifications: from ChIP-Seq data to chromatin-signaling. Nucleic Acids Res 2014; 42:13689-95.

22. Kent WJ, Sugnet CW, Furey TS, et al. The human genome browser at UCSC. Genome Res 2002; 12:996-1006.

23. Morgan M, Falcon S, Gentleman R. GSEABase: Gene set enrichment data structures and methods. R package. Version 1.30.2. Seattle, USA: Bioconductor.org, Fred Hutchinson Cancer Research Center.

24. Du P, Kibbe WA, Lin SM. lumi: a pipeline for processing Illumina microarray. Bioinformatics 2008; 24:1547-8. 
25. Ritchie ME, Phipson B, Wu D, et al. limma powers differential expression analyses for RNA-sequencing and microarray studies. Nucleic Acids Res 2015; 43:e47.

26. Matys V, Kel-Margoulis OV, Fricke E, et al. TRANSFAC and its module TRANS Compel: transcriptional gene regulation in eukaryotes. Nucleic Acids Res 2006; 34 : D108-10.

27. Turatsinze JV, Thomas-Chollier M, Defrance M, van Helden J. Using RSAT to scan genome sequences for transcription factor binding sites and cis-regulatory modules. Nat Protoc 2008; 3:1578-88.

28. Kielbasa SM, Klein H, Roider HG, Vingron M, Bluthgen N. TransFind-predicting transcriptional regulators for gene sets. Nucleic Acids Res2010; 38:W275-80.

29. Monroe KM, McWhirter SM, Vance RE. Identification of host cytosolic sensors and bacterial factors regulating the type I interferon response to Legionella pneumophila. PLoS Pathog 2009; 5:e1000665.

30. Schmeck B, N'Guessan PD, Ollomang M, et al. Legionella pneumophila-induced NF-kappaB- and MAPK-dependent cytokine release by lung epithelial cells. Eur Respir J 2007; 29:25-33.
31. Hippenstiel S, Opitz B, Schmeck B, Suttorp N. Lung epithelium as a sentinel and effector system in pneumonia-molecular mechanisms of pathogen recognition and signal transduction. Respir Res 2006; 7:97.

32. Jensen LJ, Kuhn M, Stark M, et al. STRING 8-a global view on proteins and their functional interactions in 630 organisms. Nucleic Acids Res 2009; 37:D412-6.

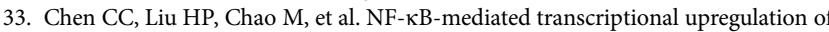
TNFAIP2 by the Epstein-Barr virus oncoprotein, LMP1, promotes cell motility in nasopharyngeal carcinoma. Oncogene 2014; 33:3648-59.

34. Price CT, Abu Kwaik Y. The transcriptome of Legionella pneumophila-infected human monocyte-derived macrophages. PLoS One 2014; 9:e114914.

35. Lleres D, Denegri M, Biggiogera M, Ajuh P, Lamond AI. Direct interaction be tween hnRNP-M and CDC5L/PLRG1 proteins affects alternative splice site choice. EMBO Rep 2010; 11:445-51.

36. Bodemann BO, Orvedahl A, Cheng T, et al. RalB and the exocyst mediate the cellular starvation response by direct activation of autophagosome assembly. Cell 2011; 144:253-67. 